\title{
Imaging diagnosis of extrahepatic biliary tract obstruction with acquired portosystemic shunt in a cat
}

\author{
Tae-Sung Hwang, Won-Seok Jang, Young-Min Yoon, Dong-In Jung, Hee Chun Lee* \\ Institute of Animal Medicine, College of Veterinary Medicine, Gyeongsang National University, Jinju 52828, Korea
}

(Received: November 14, 2018; Revised: December 10, 2018; Accepted: December 18, 2018)

\begin{abstract}
A 2-year-old, spayed female, Korean domestic short-hair cat was presented with depression and vomiting. The patient had history of weight loss lasting seven months. Physical examination revealed icterus in the pinna, oral mucosa, and sclera. Based on ultrasonography and computed tomography, tentative diagnosis was extrahepatic biliary tract obstruction with acquired portosystemic shunt (PSS). Tumor or inflammation of hepatobiliary system was suspected as the cause of obstruction of the common bile duct. But it could not be determined without biopsy. The severely dilated cystic duct was considered to cause portal hypertension and secondary multiple PSS. The patient expired without histopathologic examination.
\end{abstract}

Keywords: computed tomography, extrahepatic cholestasis, portasystemic shunt, ultrasonography

Extrahepatic biliary tract obstruction (EHBO) is caused by obstruction of extrahepatic biliary system flowing into the duodenum. Unlike dogs, EHBO is not a common disease and underlying causes are not well known in cats [7, 12]. However, EHBO occurs after extraluminal compression or intraluminal obstruction of the common bile duct. Its underlying causes can be tumors or inflammatory changes of hepatobiliary system, adjacent organs and lymph nodes [4]. Additionally, choledocholithiasis, congenital disorders such as polycystic kidney diseases diaphragmatic hernia and parasitic infection also can be causes of EHBO. Biliary cirrhosis, portal hypertension and acquired portosystemic shunt (PSS) may occur due to chronicity [4].

Computed tomography (CT) can reveal dilated biliary tract and presence of tumors or inflammation of hepatobiliary system, adjacent organs and lymph nodes after administration of contrast medium [14]. In case of acquired PSS, as a result of portal hypertension, variable types of multiple small tortuous vessels can be detected between portal and systemic circulation on CT. Vessels are mainly found between the renal vein and splenic vein and enter caudal vena cava directly or its tributaries [1]. This report describes imaging features of EHBO with acquired PSS.

A two-year-old, spayed female, Korean domestic short-hair cat was presented with history of depression, vomiting and weight loss lasting seven months. Physical examination revealed icterus in the pinna, oral mucosa, and sclera. Abdominal mass in the right cranial abdomen was also iden- tified on palpation. Concentrations of alkaline phosphatase (385 U/L, reference range 10 to $90 \mathrm{U} / \mathrm{L}$ ), alanine transaminase $(167 \mathrm{U} / \mathrm{L}$, reference range 20 to $100 \mathrm{U} / \mathrm{L})$, gammaglutamyl transferase ( $35 \mathrm{U} / \mathrm{L}$, reference range 0 to $2 \mathrm{U} / \mathrm{L})$, bile acid $(65 \mu \mathrm{mol} / \mathrm{L}$, reference range $65 \mu \mathrm{mol} / \mathrm{L})$, total bilirubin $(2.1 \mathrm{mg} / \mathrm{dL}$, reference range 0.1 to $1.6 \mathrm{mg} / \mathrm{dL})$ and ammonia $(104 \mu \mathrm{mol} / \mathrm{L}$, reference range 0 to $95 \mu \mathrm{mol} / \mathrm{L})$ increased in serum chemistry values. Leukocytosis $(23.61 \times$ $10^{9} / \mathrm{L}$, reference range 2.87 to $17.02 \times 10^{9} / \mathrm{L}$ ) was also identified in a complete blood cell count. Abdominal radiography reveals a large soft tissue mass in cranial abdominal cavity. Abdominal mass caused left lateral displacement of stomach and caudal displacement of bilateral kidney.

Abdominal ultrasonography revealed severely dilated biliary system including intrahepatic duct and cystic duct. Because of severely dilated cystic duct, the common bile duct cannot be evaluated. Echogenic sludge in gallbladder and irregularly thickened gallbladder wall were identified. Tortuous vessels surrounding the urinary bladder were detected and they were thought to be originated from the spleen. A large amount of peritoneal effusion was also identified. There were no remarkable findings in the pancreas (Fig. 1).

On CT and contrast enhanced CT scan, there was mildly heterogeneous enhancement of hepatic parenchyma with irregular contour. Severe dilations of intrahepatic duct, cystic duct and proximal region of common bile duct were identified. Focal thickening and contrast enhancement of distal common bile duct wall were also observed (Fig. 2). Addi-

*Corresponding author

Tel: +82-55-772-2356, Fax: +82-55-772-2330

E-mail: 1hc@gnu.ac.kr 

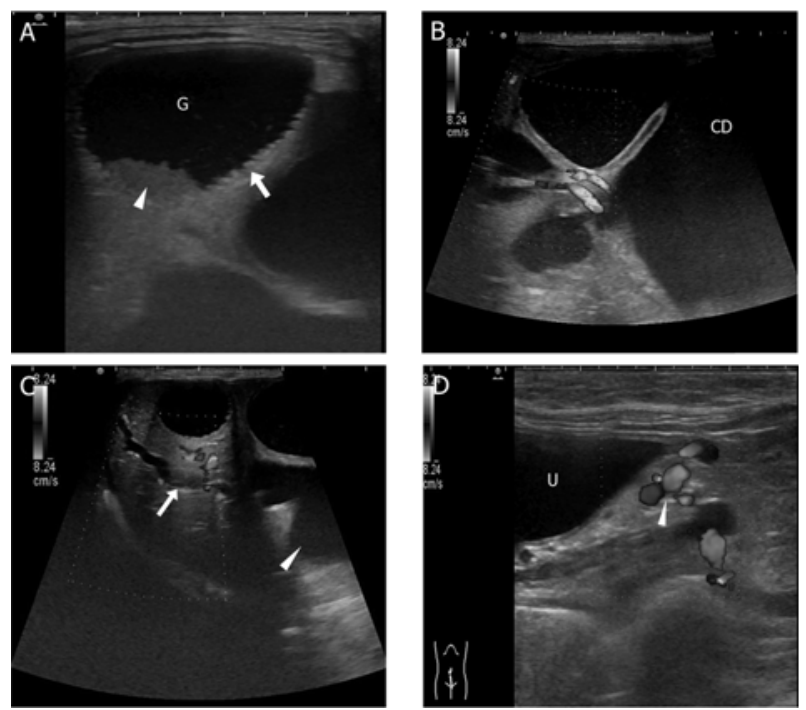

Fig. 1. Abdominal ultrasonographic images of the cat. (A) Gallbladder with irregularly thickened wall (arrow) is found. Gravity-dependent echogenic sludge (arrowhead) is also identified. (B) Severely dilated cystic duct is observed. (C) Severely and tortuously dilated intrahepatic duct (arrow) and anechoic free fluid (arrowhead) are identified. (D) Tortuous vessels (arrow head) around urinary bladder were found. They were thought to be originated from the spleen. $\mathrm{G}$, gallbladder; $\mathrm{CD}$, cystic duct; $\mathrm{U}$, urinary bladder.
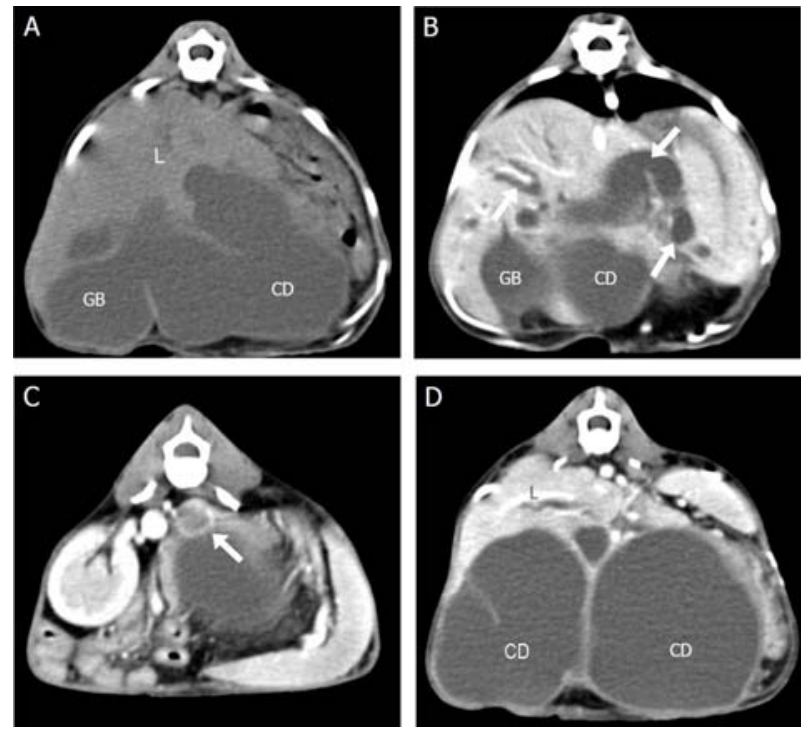

Fig. 2. Transverse view of precontrast (A) and contrast enhanced (B-D) computed tomography images of the cat. (A) Severely dilated cystic duct (CD) is present. Mildly irregular contour of the liver (L) is also identified. (B) Dilated intrahepatic ducts (arrows) are observed. (C) Focal thickening and contrast enhancement of distal common bile duct wall (arrow) are identified. (D) During the portal phase, mildly heterogeneous enhanced hepatic parenchyma with irregular contour is found. Severely dilated CD is also identified. GB, gallbladder.
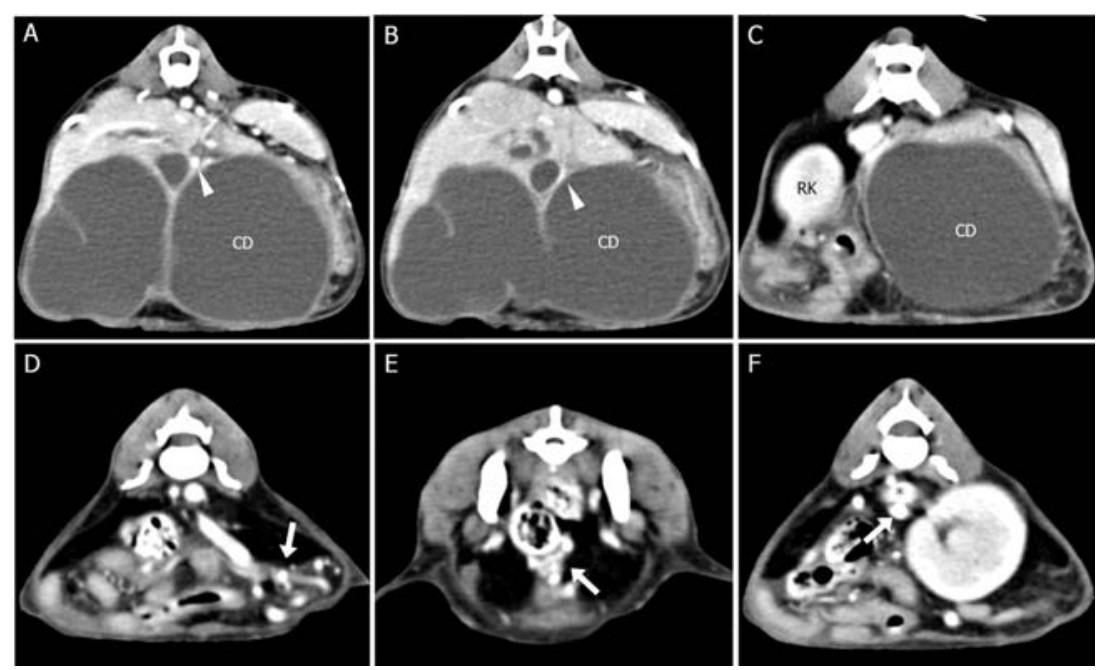

Fig. 3. Transverse view of contrast enhanced computed tomography images of the cat. (A and B) Portal vein (arrowheads) compressed by dilated cystic duct is observed. (C) At the level of right kidney, peritoneal effusion is detected in the right ventral abdominal cavity. (D-F) Varicose veins (arrows) are identified between spleen and descending colon. The vessels were originated from the spleen. It is also found that the vessels are entering caudal vena cava (asterisk) at the level of descending colon. RK, right kidney.

tionally, portal vein compressed by dilated cystic duct and peritoneal effusion was identified. Varicose veins originated from the spleen were also detected. They entered caudal vena cava at the level of distal descending colon (Fig. 3).

Based on ultrasonography and CT, the tentative diagnosis was EHBO with acquired PSS. Tumors or inflammation of hepatobiliary system were suspected as the cause of obstruction of common bile duct. Because biopsy was not performed, causes of EHBO could not be determined. We considered that the severely dilated cystic duct caused portal hypertension and acquired PSS by compressing portal vein. After imaging diagnosis, the patient was given symptomatic 
treatment for two weeks in a local animal hospital. However, the patient expired without histopathologic diagnosis or necropsy.

Clinical signs of EHBO are not specific. Lethargy, intermittent fever, jaundice and vomiting can occur. With chronicity, it may lead to non-regenerative anemia and coagulopathies [4]. In a previous study, it was revealed that cats with EHBO secondary to cholelithiasis have shorter duration of clinical signs than EHBO secondary to tumor or inflammation [7]. Additionally, cats with EHBO resulting from neoplasia have significantly shorter median survival time than inflammatory disease [3]. In this case, the patient had history of depression, vomiting and weight loss lasting seven months. The patient expired after imaging diagnosis and symptomatic treatment for two weeks.

Radiographic features of EHBO are also non-specific [4, 12]. This case revealed abdominal mass in cranial abdominal cavity. However, it could not be diagnosed as EHBO because of these non-specific features.

In normal cats, intrahepatic and extrahepatic ducts are not visible on ultrasonography. So visualization of both ducts can be thought of as dilation of the biliary system [6, 11]. In addition, common bile duct can be dilated more than $5 \mathrm{~mm}$ in diameter [11]. Other ultrasonographic findings suggest that inflammation or neoplasia of hepatobiliary system and adjacent organs can be observed in cats with EHBO [4]. In this case, severely dilated cystic duct and tortuously dilated intrahepatic duct were detected on ultrasonography. Irregularly thickened gallbladder wall and echogenic sludge in gallbladder were thought as inflammatory change of hepatobiliary system.

Although the underlying cause of EHBO is not fully known in cats, the most common causes are inflammation or tumor of hepatobiliary system and pancreas $[12,15]$. In this case, there were no remarkable abnormalities in other organs adjacent to hepatobiliary system on ultrasonography and CT. Choledocholelith was not also identified. On contrast enhanced $\mathrm{CT}$, focal wall thickening in the distal common bile duct with contrast enhancement was detected, suggesting possibility of inflammation of the biliary tract.

In humans, contrast-enhanced CT findings in malignant biliary stricture have been reported. It is likely to be a malignant common bile duct stricture if the affected bile duct wall is focally thickened more than $1.5 \mathrm{~mm}$ and strongly contrastenhanced in arterial phase and portal phase [5]. In this case, focal thickening of distal common bile duct wall was detected, and thickness was $2.1 \mathrm{~mm}$ on contrast-enhanced CT. Additionally, strong enhancement of the thickened common bile duct wall was also observed in the portal phase. Therefore, inflammation or tumor of hepatobiliary system was suspected to induce the EHBO of this case. However, it was not possible to differentiate the two diseases just only based on clinical and imaging findings.

Although EHBO can result from cholangiohepatitis in cats, cholangiohepatitis can be secondary to EHBO [12, 15]. In this case, ultrasonographic and computed tomographic findings suggest that cholangiohepatitis was identified. However, with these findings, it was unclear if hepatobiliary inflammation is the primary cause of EHBO or secondary to EHBO.

The degree of biliary tract dilation cannot differentiate the cause of EHBO on ultrasonography [7]. In this case, the severely dilated biliary tract is due to chronicity of obstruction or irreversible loss of elasticity of biliary tract [9].

In dogs, acquired PSS mainly results from regenerative nodules and portal hypertension associated with cirrhosis [8, 13]. Conversely, cats rarely develop portal hypertension secondary to cirrhosis. Hence, cats with acquired PSS have been thought to be rare [10]. Portal hypertension can be classified into three categories: prehepatic, intrahepatic and posthepatic. Prehepatic portal hypertension is caused by intraluminal obstruction or extraluminal compression of portal vein. Portal hypertension secondary to cirrhosis corresponds to intrahepatic portal hypertension [2]. In this case, regenerative nodules indicating cirrhosis were not detected on ultrasonography and compression of portal vein due to severely dilated cystic duct was identified on CT. Possibly acquired PSS resulted from prehepatic portal hypertension.

Limitation of this case was that the histopathologic examination or necropsy was not performed.

In this case, EHBO was tentatively diagnosed by using ultrasonography and CT without histopathologic examination and necropsy. If further studies about computed tomographic features of extrahepatic biliary duct for differentiating malignant from benign EHBO are performed, usefulness of $\mathrm{CT}$ in predicting causes of EHBO will increase.

\section{References}

1. Bertolini G. Acquired portal collateral circulation in the dog and cat. Vet Radiol Ultrasound 2010, 51, 25-33.

2. Buob S, Johnston AN, Webster CRL. Portal hypertension: pathophysiology, diagnosis, and treatment. J Vet Intern Med 2011, 25, 169-186.

3. Buote NJ, Mitchell SL, Penninck D, Freeman LM, Webster CRL. Cholecystoenterostomy for treatment of extrahepatic biliary tract obstruction in cats: 22 cases (19942003). J Am Vet Med Assoc 2006, 228, 1376-1382.

4. Center SA. Diseases of the gallbladder and biliary tree. Vet Clin North Am Small Anim Pract 2009, 39, 543-598.

5. Choi SH, Han JK, Lee JM, Lee KH, Kim SH, Lee JY, Choi BI. Differentiating malignant from benign common bile duct stricture with multiphasic helical CT. Radiology 2005, 236, 178-183.

6. Cornell KK, Jakovljevic S, Waters DJ, Prostredny J, Salisbury SK, DeNicola DB. Extrahepatic biliary obstruction secondary to diaphragmatic hernia in two cats. J Am Anim Hosp Assoc 1993, 29, 502-507.

7. Gaillot HA, Penninck DG, Webster CRL, Crawford S. Ultrasonographic features of extrahepatic biliary obstruction in 30 cats. Vet Radiol Ultrasound 2007, 48, 439-437.

8. Johnson SE. Portal hypertension. Part I. Pathophysiology and 
clinical consequences. Compend Contin Educ Pract Vet 1987, 9, 741-748

9. Laing FC, Jeffrey RB Jr, Wing VW, Nyberg DA. Biliary dilatation: defining the level and cause by real-time US. Radiology 1986, 160, 39-42.

10. Langdon P, Cohn LA, Kreeger JM, Priddy NH. Acquired portosystemic shunting in two cats. J Am Anim Hosp Assoc 2002, 38, 21-27

11. Léveillé R, Biller DS, Shiroma JT. Sonographic evaluation of the common bile duct in cats. J Vet Intern Med 1996, 10, 296-299.
12. Mayhew PD, Holt DE, McLear RC, Washabau RJ. Pathogenesis and outcome of extrahepatic biliary obstruction in cats. J Small Anim Pract 2002, 43, 247-253.

13. Williams EJ, Iredale JP. Liver cirrhosis. Postgrad Med J 1998, 74, 193-202.

14. Wisner ER, Zwingenberger AL. Atlas of Small Animal CT and MRI. pp. 522-536, Wiley Blackwell, Ames, 2015.

15. Zawie DA, Shaker E. Diseases of the liver. In: Sherding RG (ed.). The Cat. Diseases and Clinical Management. pp. 1015-1036, Churchill Livingstone, New York, 1989. 